\title{
Influence of METHoxyflurane on ANtiplatelet Effect of ticagrelor in patients with unstable angina pectoris: Rationale and a protocol of a randomized clinical METHANE-SIRIO 4 study
}

\author{
Piotr Niezgoda ${ }^{1}$ (D) Malwina Barańska ${ }^{1}$, Piotr Adamski ${ }^{1}$, Łukasz Pietrzykowski², \\ Michał Piotr Marszałł ${ }^{1}$, Wojciech Wojakowski ${ }^{3}$, Wiktor Kuliczkowski ${ }^{4}$, Diana Gorog ${ }^{5}$, \\ Bernd Jilma $^{6}$, Klaudiusz Nadolny ${ }^{7,8}$, Eliano Pio Navarese ${ }^{1}$, Aldona Kubica ${ }^{2}$, Jacek Kubica ${ }^{1}$ \\ ${ }^{1}$ Collegium Medicum, Nicolaus Copernicus University, Bydgoszcz, Poland \\ ${ }^{2}$ Department of Health Promotion, Collegium Medicum, Nicolaus Copernicus University, Bydgoszcz, Poland \\ ${ }^{3}$ Medical University of Silesia, Katowice, Poland \\ ${ }^{4}$ University and Clinical Hospital of Wroclaw, Poland \\ ${ }^{5}$ National Heart and Lung Institute, Imperial College London, United Kingdom \\ ${ }^{6}$ Department of Clinical Pharmacology, Medical University of Vienna, Austria \\ ${ }^{7}$ Medical University of Bialystok, Poland \\ ${ }^{8}$ Higher School of Strategic Planning, Dabrowa Gornicza, Poland
}

\section{Introduction}

Results of the IMPRESSION trial published in 2015 proved that morphine use in patients with acute coronary syndromes (ACS) is associated with an undesirable impact on pharmacokinetics (PK) and pharmacodynamics (PD) of ticagrelor [1]. Several other studies of the antiplatelet activity of all oral $\mathrm{P}_{2} \mathrm{Y}_{12}$ receptor inhibitors, ticagrelor, prasugrel and clopidogrel, also reported noticeable impairment of their antiaggregatory effect when morphine was co-administered in the course of ACS or in healthy volunteers [2-5]. This negative effect may be substantially increased by medical approaches such as mild therapeutic hypothermia in patients presenting with out of hospital cardiac arrest [6]. Based on available data, the latest European Society of Cardiology (ESC) guidelines on the management of acute ST-segment elevation myocardial infarction patients reduced the class of recommendation of titrated opioid use to IIa [7].
Despite this, morphine is still a standard analgesic treatment in ACS patients and it should not be routinely withdrawn [8-10]. Based on available research, morphine, acting via $\mu$-opioid receptors, was found to inhibit gastrointestinal motility or induce adverse effects such as nausea or vomiting [11]. Therefore, an inevitable consequence of those clinical trials was to establish an approach which would maximally allow overcoming the so-called "morphine-effect" as efficient pretreatment with P2 $\mathrm{Y}_{12}$ inhibitors being proven to be beneficial [12]. Crushing $\mathrm{P}_{2} \mathrm{Y}_{12}$ receptor inhibitors tablets was consistently found to be successful in terms of improvement of PK/PD of these medications [13-15]. As was found in one of our previous trials, the addition of metoclopramide, which may to some extent positively influence the PK/PD of ticagrelor, but such an approach definitely cannot be considered as a method of overcoming the "morphine effect" [16]. On the other hand, the addition of naloxone was not beneficial in terms of PK/PD profile of

Address for correspondence: Dr. Piotr Niezgoda, Collegium Medicum, Nicolaus Copernicus University, ul. M. Skłodowskiej-Curie 9, 85-094 Bydgoszcz, Poland, tel: +48 52585 4023, fax: + 48525854024 , e-mail: piotr.niezgoda1986@gmail.com

Received: 9.06.2021 Accepted: 22.08.2021 Early publication date: 8.10.2021

This article is available in open access under Creative Common Attribution-Non-Commercial-No Derivatives 4.0 International (CC BY-NC-ND 4.0) license, allowing to download articles and share them with others as long as they credit the authors and the publisher, but without permission to change them in any way or use them commercially. 
ticagrelor [17]. Also, switching from morphine to another opioid analgesic, fentanyl, did not allow to waive the negative morphine effect in the PACIFY trial [18]. It was decided to design a clinical study aiming to evaluate the impact of methoxyflurane on the PD of ticagrelor in patients diagnosed with unstable angina pectoris (UA). Methoxyflurane is an inhaled anesthetic, registered in Poland in emergency medicine for pain alleviation in trauma patients. The drug was widely used in the 1960s to induce general anesthesia, however its clinical utility was reduced with the development of novel anesthetic agents. Taking into account its different mechanism of action, it can be presumed that, contrary to morphine, no respiratory depression should be observed as well as no attenuation or delay of antiaggregatory effect of ticagrelor should occur, as no interaction with the $\mu$-receptor in the gastrointestinal tract is related to activity of methoxyflurane.

\section{Methods}

This study was designed as a phase IV, open-label, randomized, multi-center clinical trial. The protocol of the study was approved by the Ethics Committee of The Nicolaus Copernicus University and received approval number KB 37/2020. All UA patients admitted to the study centers will be screened for eligibility. UA is defined as myocardial ischemia symptoms in class III or IV according to the Canadian Cardiovascular Society (CCS) with troponin serum concentration within the reference range accompanied with newly diagnosed regional impairment of left ventricle contraction in echocardiography and/or acute signs of ischemia in electrocardiogram at admission. Major inclusion criteria include males and non-pregnant females, aged 18-80, diagnosed with UA, planned for coronary angiography and percutaneous coronary intervention if required, and graded $<140$ points in GRACE score. Among the exclusion criteria current use of oral anticoagulants, $\mathrm{P} 2 \mathrm{Y}_{12}$ receptor inhibitors, heparin, history of coagulation disorders, intracranial bleeding or recent gastrointestinal hemorrhage as well as clinically-significant bradycardia or $2^{\text {nd }} / 3^{\text {rd }}$ degree atrioventricular block are also to be noted. The complete list of study inclusion and exclusion criteria is presented in Table 1. All study-related procedures will be conducted in full accordance with The Declaration of Helsinki and Good Clinical Practice regulations after the obtainment of written informed consent from each enrolled participant. Patients will be randomized in a 1:1:1 ratio into
Table 1. A complete list of inclusion and exclusion criteria in the study.

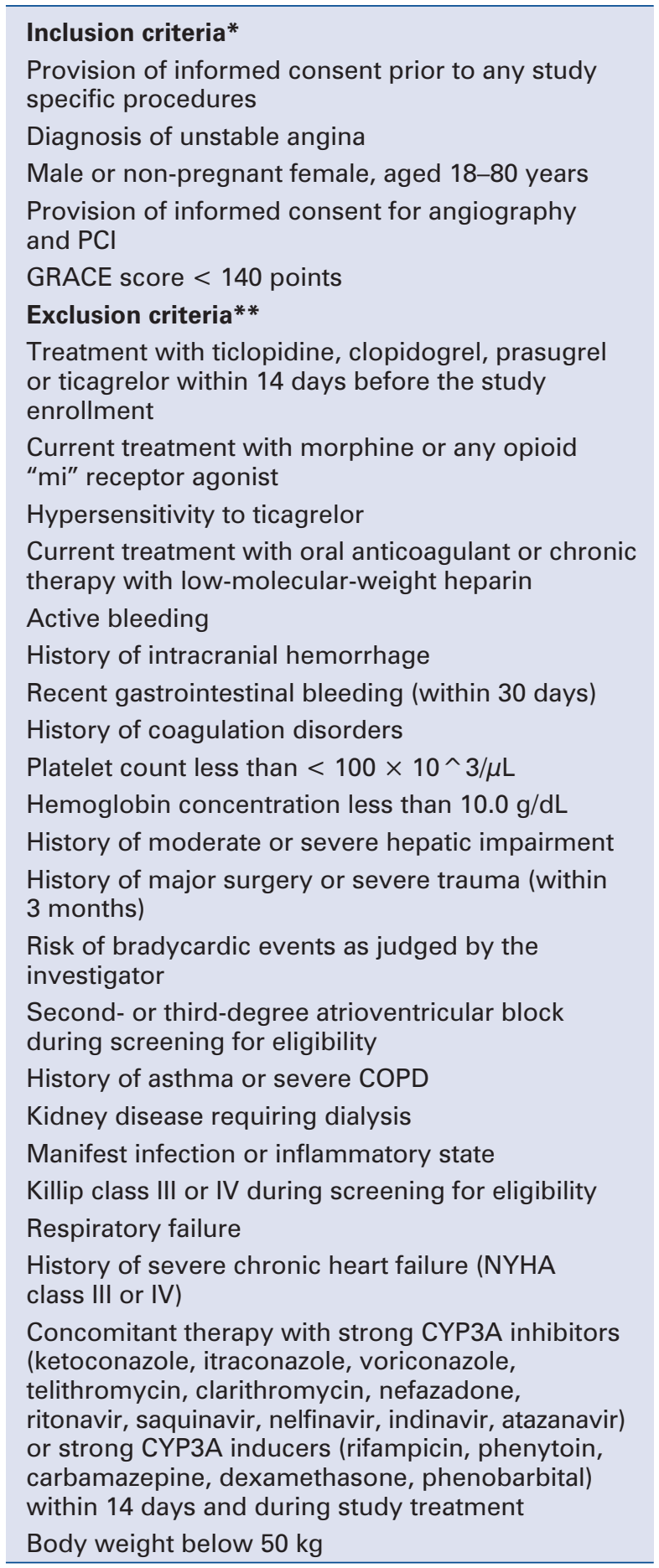

${ }^{*}$ All criteria must be met; ${ }^{*}$ None of the criteria can be met; COPD - chronic obstructive pulmonary disease; NYHA — New York Heart association; $\mathrm{PCl}$ - percutaneous coronary intervention

the study arms as follows: 1) $180 \mathrm{mg}$ ticagrelor (2 integral tablets of $90 \mathrm{mg}$ ticagrelor) followed by $3 \mathrm{mg}$ inhaled methoxyflurane methoxyflurane (3 $\mathrm{mg}$ is a dose included in a single-use dispenser, 


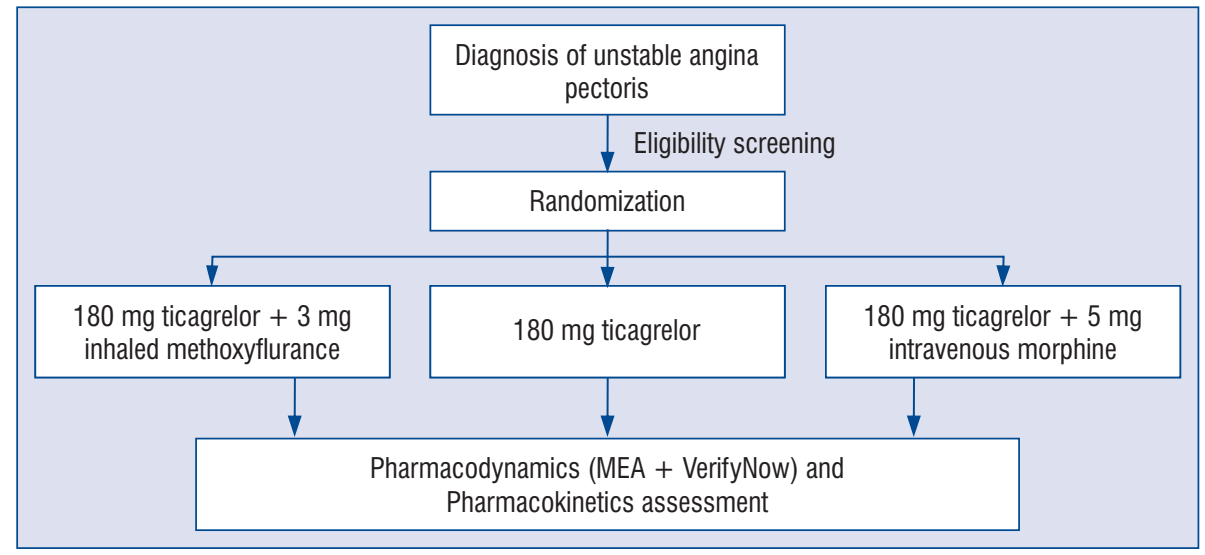

Figure 1. The patients' flow through the study; MEA - multiple electrode aggregometry.

the patient will be advised to inhale the drug for 15 minutes between time-points: baseline and 15 minutes post ticagrelor loading dose [LD]), 2) $180 \mathrm{mg}$ ticagrelor followed by $5 \mathrm{mg}$ intravenous morphine, 3) $180 \mathrm{mg}$ ticagrelor alone. Each study participant will undergo pharmacodynamic assessment at time points 0 (prior to the administration of ticagrelor LD) and at 15, 30, 45, 60, 120, 180, 240, 360 minutes after the LD. Platelet activity will be assessed using multiple electrode aggregometry (MEA). Pharmacokinetic assessment of ticagrelor and its active metabolite will be performed at each time point. The schematic presentation of the patients' flow through the study is presented in Figure 1.

\section{Sample size calculation}

Taking into account previous studies conducted in the Department of Cardiology in Bydgoszcz, Poland, which aimed to evaluate pharmacokinetics and pharmacodynamics of ticagrelor in patients treated for UA, as well as the fact that no similar clinical studies were completed to date, a group of 75 patients, 25 for each study arm was estimated as sufficient for the obtainment of significant differences between the measured parameters.

\section{Blood sample collection}

Directly after randomization, all study participants will undergo blood sampling collection (time point 0 ), which will be followed by the administration of ticagrelor LD and analgesics according to the study protocol. The remaining blood samples will be obtained at the following time points: 15,30 , $45,60,120,180,240,360$ minutes after ticagrelor LD by nurses employed in each study site. The use of vascular catheters is planned, which is meant to minimize the potential patient discomfort associated with blood sample obtainment. The procedure will be supervised by doctors responsible for the recruitment of participants.

\section{Endpoints}

The primary endpoint of the trial is the mean platelet reactivity between the study arms, assessed using the Multiplate Analyzer within the time frame of 6 hours following ticagrelor LD.

Secondary endpoints of the study were defined as (1) the percentage of patients with high platelet reactivity throughout the study period and (2) mean time to achieve platelet reactivity below the threshold for high platelet reactivity; (3) area under the plasma concentration-time curve for ticagrelor and its active metabolite between the study arms.

\section{Pharmacodynamics and pharmacokinetics assessment}

The evaluation of platelet reactivity is planned for all study participants at the aforementioned study time points using MEA. Platelet reactivity testing will be performed in the internal research laboratories by investigators employed at each center conducting the study-related procedures.

According to the study protocol, the assessment of pharmacokinetic parameters of ticagrelor and its active metabolite for all enrolled patients will be performed at each study time point. All samples will be evaluated in the Study Central Lab.

\section{Safety assessment}

Each study participant will be treated according to the latest ESC guidelines for the manage- 
ment of patients presenting with ACS, which will include coronary angiography and percutaneous coronary intervention, if necessary. All enrolled patients will receive a complete pharmacological treatment for ACS comprised of dual antiplatelet therapy, statin, beta-blocker and angiotensin-converting enzyme inhibitor or angiotensin II receptor blocker, unless contraindicated. Only subjects with $<140$ points according to GRACE score will be enrolled, which will allow the investigators to postpone coronary angiography for at least 6 hours after the randomization; the time which is required to complete the blood sampling scheme. In case of any episode of severe pain with no improvement after the baseline administration of analgesic agents according to randomization, an additional dose of $3 \mathrm{mg}$ of inhaled methoxyflurane or $5 \mathrm{mg}$ of intravenous morphine will be given appropriately. The evaluation of drug efficacy in such cases will be performed every 3 minutes. However, any case of unbearable persisting chest pain will result in the premature termination of the patient's participation in the trial. The patient will undergo immediate coronary angiography and will receive symptoms-driven analgesia. Any adverse effects of administered medications, including abnormalities in patients' vital signs, blood pressure, heart rate, respiratory rate, arrhythmias, dyspnea as well as deterioration of kidney or hepatic function based on laboratory tests performed routinely during hospitalization, will be recorded and treated appropriately as to the event.

\section{Discussion}

Based on the results of several worldwide clinical trials mentioned above in this manuscript, it has become clear that morphine use is associated with undesirable effects on PK and PD of antiplatelet agents. Nevertheless, none of the methods of overcoming the so-called "morphine effect" including crushing $\mathrm{P}_{2} \mathrm{Y}_{12}$ receptor inhibitors tablets, sublingual administration of those agents or co-administration of either metoclopramide or naloxone turned out efficient enough to ensure rapid platelet inhibition which is crucial to succeed in the treatment of ACS. Moreover, changing morphine to another opioid agent, fentanyl, also showed no superiority in terms of improvement of $\mathrm{PK} / \mathrm{PD}$ profile of ticagrelor, which may be explained by the class effect of those drugs. To date, the clinical utility of methoxyflurane in ACS patients has never been examined. It was decided to exclusively select UA patients so as make this trial maximally comparable to previously conducted studies aiming to evaluate the influence of various ticagrelor administration strategies on its $\mathrm{PK} / \mathrm{PD}$ profile [13, $16,17]$. However, taking into account differences in the mechanism of action of methoxyflurane and opioids, no impairment of ticagrelor pharmacodynamic profile may be expected. If such a thesis is confirmed, it may lead to a major breakthrough in terms of effective antiplatelet treatment of patients presenting with ACS.

\section{Trial status}

The study was registered on clinicaltrials. gov and received the registration number NCT04442919, date of registration 27 June 2020. It is currently recruiting participants. https://clinicaltrials.gov/ct2/show/NCT04442919.

\section{Ethics approval and consent to participate}

The protocol of the study was approved by the Ethics Committee of The Nicolaus Copernicus University and received approval number KB 37/2020.

\section{Funding}

This research received financial support from the Nicolaus Copernicus University, Poland, through IDUB Project (Emerging Field - Metabolic Civilization Diseases).

\section{Acknowledgments}

The authors would like to thank all doctors and nurses of the Department of Cardiology who contributed to this project for their invaluable involvement which makes conducting this clinical trial possible.

Conflict of interest: Klaudiusz Nadolny delivered lectures for AstraZeneca. All remaining authors declare no conflict of interest.

\section{References}

1. Kubica J, Adamski P, Ostrowska M, et al. Morphine delays and attenuates ticagrelor exposure and action in patients with myocardial infarction: the randomized, double-blind, placebo-controlled IMPRESSION trial. Eur Heart J. 2015; 37(3): 245-252, doi: 10.1093/eurheartj/ehv547.

2. Hobl EL, Reiter B, Schoergenhofer C, et al. Morphine decreases ticagrelor concentrations but not its antiplatelet effects: a randomized trial in healthy volunteers. Eur J Clin Invest. 2016; 46(1): 7-14, doi: 10.1111/eci.12550, indexed in Pubmed: 26449338.

3. Parodi G, Bellandi B, Xanthopoulou I, et al. Morphine is associated with a delayed activity of oral antiplatelet agents in patients with ST-elevation acute myocardial infarction undergoing primary percutaneous coronary intervention. Circ Cardiovasc Interv. 2015; 8(1), doi: 10.1161/CIRCINTERVENTIONS.114.001593, indexed in Pubmed: 25552565. 
4. Hobl EL, Stimpfl T, Ebner J, et al. Morphine decreases clopidogrel concentrations and effects: a randomized, double-blind, placebo-controlled trial. J Am Coll Cardiol. 2014; 63(7): 630-635, doi: 10.1016/j.jacc.2013.10.068, indexed in Pubmed: 24315907.

5. Hobl EL, Reiter B, Schoergenhofer C, et al. Morphine interaction with prasugrel: a double-blind, cross-over trial in healthy volunteers. Clin Res Cardiol. 2016; 105(4): 349-355, doi: 10.1007/ s00392-015-0927-z, indexed in Pubmed: 26493304.

6. Umińska JM, Ratajczak J, Buszko K, et al. Impact of mild therapeutic hypothermia on bioavailability of ticagrelor in patients with acute myocardial infarction after out-of-hospital cardiac arrest. Cardiol J. 2020; 27(6): 780-788, doi: 10.5603/CJ.a2019.0024, indexed in Pubmed: 30799546.

7. Ibanez B, James S, Agewall S, et al. et al.. ESC Scientific Document Group. 2017 ESC Guidelines for the management of acute myocardial infarction in patients presenting with ST-segment elevation: The Task Force for the management of acute myocardial infarction in patients presenting with ST-segment elevation of the European Society of Cardiology (ESC). Eur Heart J. 2018; 39(2): 119-177, doi: 10.1093/eurheartj/ehx393, indexed in Pubmed: 28886621.

8. Ostrowska M, Gorog D. Does morphine remain a standard of care in acute myocardial infarction? Med Res J. 2020; 5(1): 46-49, doi: 10.5603/mrj.a2020.0009.

9. Kubica J, Adamski P, Paciorek P, et al. Treatment of patients with acute coronary syndrome: Recommendations for medical emergency teams: Focus on antiplatelet therapies. Updated experts' standpoint. Cardiol J. 2018; 25(3): 291-300, doi: 10.5603/ CJ.a2018.0042, indexed in Pubmed: 29671864.

10. Nadolny K, Ładny JR, Gałązkowski R, et al. Medical emergency team interventions in patients with ST-segment elevation myocardial infarction in Poland in 2018. Kardiol Pol. 2020; 78(4): 292-299, doi: 10.33963/KP.15222, indexed in Pubmed: 32124868 .

11. Kubica J, Kubica A, Jilma B, et al. Impact of morphine on antiplatelet effects of oral P2Y12 receptor inhibitors. Int J Cardiol.
2016; 215: 201-208, doi: 10.1016/j.jicard.2016.04.077, indexed in Pubmed: 27128531.

12. Zhang K, Yang W, Zhang M, et al. Pretreatment with antiplatelet drugs improves the cardiac function after myocardial infarction without reperfusion in a mouse model. Cardiol J. 2021; 28(1): 118-128, doi: 10.5603/CJ.a2019.0051, indexed in Pubmed: 31106840 .

13. Niezgoda P, Sikora J, Barańska M, et al. Crushed sublingual versus oral ticagrelor administration strategies in patients with unstable angina. A pharmacokinetic/pharmacodynamic study. Thromb Haemost. 2017; 117(4): 718-726, doi: 10.1160/TH1608-0670, indexed in Pubmed: 28203684.

14. Zafar MU, Farkouh ME, Fuster V, et al. Crushed clopidogrel administered via nasogastric tube has faster and greater absorption than oral whole tablets. J Interv Cardiol. 2009; 22(4): 385-389, doi: 10.1111/j.1540-8183.2009.00475.x, indexed in Pubmed: 19496900.

15. Parodi G, Xanthopoulou I, Bellandi B, et al. Ticagrelor crushed tablets administration in STEMI patients: the MOJITO study. J Am Coll Cardiol. 2015; 65(5): 511-512, doi: 10.1016/j. jacc.2014.08.056, indexed in Pubmed: 25660931.

16. Sikora J, Niezgoda P, Barańska M, et al. METoclopramide Administration as a Strategy to Overcome MORPHine-ticagrelOr Interaction in PatientS with Unstable Angina PectorIS-The METAMORPHOSIS Trial. Thromb Haemost. 2018; 118(12): 2126-2133, doi: 10.1055/s-0038-1675605, indexed in Pubmed: 30453344 .

17. Niezgoda P, Barańska MA, Sikora J, et al. Oral NAloxone to overcome the moRphine effect in acute COronary syndrome patients treated with TICagrelor - NARCOTIC trial. Cardiol J. 2020 [Epub ahead of print], doi: 10.5603/CJ.a2020.0040, indexed in Pubmed: 32207836.

18. Ibrahim K, Shah R, Goli RR, et al. Fentanyl Delays the Platelet Inhibition Effects of Oral Ticagrelor: Full Report of the PACIFY Randomized Clinical Trial. Thromb Haemost. 2018; 118(8): 1409-1418, doi: 10.1055/s-0038-1666862, indexed in Pubmed: 29972861. 